\title{
GEOMATIC CONTRIBUTION FOR THE RESTORATION PROJECT OF THE VALENTINO CASTLE GREEN ROOM. FROM DATA ACQUISITION TO INTEGRATED DOCUMENTATION
}

\author{
G. Patrucco ${ }^{1, *}$, F. Chiabrando ${ }^{1}$, A. Dameri ${ }^{1}$, L. Teppati Losè ${ }^{1}$ \\ ${ }^{1}$ Department of Architecture and Design (DAD) - Politecnico di Torino, Viale Mattioli 39, 10125 Torino (Italy) \\ (giacomo.patrucco, filiberto.chiabrando, annalisa.dameri, lorenzo.teppati)@ polito.it
}

Commission II, WG II/8

KEY WORDS: Laser scanning, Point clouds, 3D model, Built Heritage documentation, Hyperspectral images, Restoration

\begin{abstract}
:
In the framework of restoration operations, valuable assistance can be supplied from innovative techniques and methods developed in the field of Geomatics. Over the years, this continuous collaboration has produced synergistic and interdisciplinary results that have been successfully contributing to heritage conservation and valorisation. In the case of the current research, thorough multisensory investigations have been performed in order to provide a deeper knowledge of the Green Room of the Valentino Castle in Turin and to support the planning of the future restoration works that will involve this valuable asset. In the framework of this experience, four LiDAR systems have been employed in order to evaluate the different results obtainable from the sensors. Additionally, a complete photogrammetric close-range survey has been carried out, and some tests were completed using a hyperspectral camera. The workflow followed during the current research is described in this paper, and a comparison between the obtained outputs is proposed, focusing on the characteristics of these metric products, useful and sometimes necessary in the framework of the restoration project. Besides, some considerations on the advantages and the issues connected with the use of these reality-based data as a starting point for HBIM (Heritage Building Information Modeling) model generation are proposed, along with some observations about the potentialities of a photogrammetric co-registration approach using spectrum technologies for deterioration/decay detection and monitoring of heritage.
\end{abstract}

\section{INTRODUCTION}

In the past years, geomatics instruments and techniques have proven themselves as valuable tools in the different phases of a restoration project (Pracchi and Barazzetti 2019). One of the primary efforts of the researchers working in this field has been related to the development of new strategies to enhance interdisciplinary and multidisciplinary collaboration between the different specialists involved in a restoration project (Beltrami et al. 2019). Moreover, the contribution of these techniques has also been evaluated for the creation of up to date communication and promotion campaigns in order to disseminate the results of a restoration project and the knowledge gained during this process.

Concerning the activities connected with a restoration project, scientific literature provides numerous examples of close collaborations between restorers and operators involved in the framework of 3D metric documentation. As well known, complete and accurate knowledge of the heritage that needs to be restored is a crucial aspect and an accurate metric survey should represent one of the pillars of the restoration project (García-León et al. 2019).

Unfortunately built heritage is continuously exposed to various types of risks: like accidents or natural and human disasters. In addition to the risks associated with the destruction of these valuable assets mentioned before, a significant threat to the objects belonging to cultural heritage is represented by ordinary and natural deterioration. Nowadays, one of the main challenges posed to researchers working in the field of heritage documentation is related to the development of new effective strategies and techniques able to provide full support to restorers without scarifying the criteria of accuracy, precision and data manageability.

According to these aims, the main methods that nowadays are able to provide valuable support in the different documentation phases preliminary to a restoration project (Girelli et al. 2019) are represented by both range-based techniques, e.g. connected with the use of LiDAR (Light Detection and Ranging) systems, and image-based approaches, involving the acquisition of digital images that are after that processed using a photogrammetric computer vision approach.

The choice of the methods and strategies to adopt in this particular framework can be influenced by several factors: the first one involves the possibility to acquire the geometrical features of the spaces that need to be documented, to provide the data necessary to perform the most accurate 3D model; according to the actual guideline in the restoration community this is even more related to the BIM/HBIM approach adopting parametric modelling strategies (Bacci et al. 2019; Chiabrando et al. 2016). The second one concerns the identification and representation of the decay (with manual, semi-automatic or automatic procedures) carrying out a deterioration analysis to assist the restorer's works (Bruno et al. 2019). Usually, the techniques mentioned above are integrated in order to exploit the advantage of each method for the documentation purpose.

\subsection{The case study: Valentino Castle Green Room}

The research carried out in this contribute is related to a specific room (the Green Room) located in the Noble Residential Floor of the Valentino Castle. The castle, once the royal residence of the Savoy House and now the location of the Architecture

\footnotetext{
* Corresponding author
} 
Departments of the Polytechnic University of Turin, was built and restored in several phases starting from the second half of the fifteen-century when the "casino fluviale" was acquired by the royal family in 1564 . Thanks to Cristina (daughter of the king of France) and her husband Vittorio Emanuele I essential extensions of the castle have been performed during the sixteencentury on the project of Carlo di Castellamonte (Roggero Bardelli 1992) that create the actual architectural shape of the royal residence. During the seventeen-century, a slow deterioration of the castle is reported in the historical documentation and starting from 1824 the Valentino Castle is readjusted to become a military barracks. In 1859 thanks to the Casati law the castle becomes the application school for engineers and from 1906 the "Regio Politecnico".

In this scenario, during the summer of 2019, an extensive program of knowledge began with the aim of improving the understanding of the ancient building and to develop a new restoration worksite. This objective is pursued thanks to the different expertise that are present among the researchers working at the Politecnico; it was possible to establish a multidisciplinary and interdisciplinary team that will work in order to adopt all the available cutting edges approaches form the different disciplines involved in the project. As is reported before, the main objective of this restoration works is related to the "Green Room", this description was founded in 1644 inventory where for the first time the "Green Room" is mentioned, referring to the dominant colour of the leather wall covering, "with green background and gold flowers". In contrast to other rooms where the brighter shades symbolise spring, in this room, the green is darker, some scholars have claimed it refers to the afterlife. The central fresco (Figure 1) represents the triumph of Flora-Cristina; the image takes on a funerary connotation due to the presence of the great bull, dressed as a sacrificial victim, which alludes to Vittorio Amedeo I, who was born under the zodiac sign of Taurus, and who died suddenly in 1637. In the four frescoed panels beneath the vault, the motif related to the birth of plants and flowers is described as in episodes of Ovid's Metamorphoses: the episodes take up the theme of death of the hero and his regeneration in the form of flowers. The decoration of the "Green Room" is therefore programmatic for the regency, constituting a rebirth after the death of the duke and guarantor of the duchy's independence. In the room, which was already restored in the late 1980s, the remains of the external façade of the 16th-century river villa are still visible.

\section{TESTED TERRESTRIAL LASER SCANNING SYSTEMS}

In the first part of the fieldwork, a complete range-based survey was performed using 4 different instruments to compare the characteristics and performances of each sensor.

The employed scanner and the main characteristics are reported in the following Table 1 .

\begin{tabular}{|c|c|c|c|c|}
\hline Laser scanner & $\begin{array}{c}\text { Faro Focus }^{3 \mathrm{D}} \text { X } 330 \\
\text { a }^{\frac{2}{2}}\end{array}$ & Leica BLK360 & Leica RTC360 & Riegl VZ-400i \\
\hline Distance measurements & Phase shift & Wave from digitizer & Phase shift & Time of flight \\
\hline Wavelenght & $1550 \mathrm{~nm}$ & $830 \mathrm{~nm}$ & $1550 \mathrm{~nm}$ & Near infrared \\
\hline Distance Range & $0.6-330 \mathrm{~m}$ & $0.6-60 \mathrm{~m}$ & $0.5-130 \mathrm{~m}$ & $0.5-800 \mathrm{~m}$ \\
\hline Horizontal and Vertical Range & $305 / 360^{\circ}$ & $300 / 360^{\circ}$ & $300 / 360^{\circ}$ & $100 / 360^{\circ}$ \\
\hline Distance accuracy & $\pm 2 \mathrm{~mm} @ 10 \mathrm{~m}$ & $\pm 4 \mathrm{~mm} @ 10 \mathrm{~m}$ & $\pm 1.9 \mathrm{~mm} @ 10 \mathrm{~m}$ & $\pm 5 \mathrm{~mm} @ 100 \mathrm{~m}$ \\
\hline Acquistion speed & up to $976.000 \mathrm{pt} / \mathrm{s}$ & up to $360.000 \mathrm{pt} / \mathrm{s}$ & up to $2.000 .000 \mathrm{pt} / \mathrm{s}$ & up to $500.000 \mathrm{pt} / \mathrm{s}$ \\
\hline Camera & RGB integrated & RGB+NIR integrated & RGB integrated & RGB external \\
\hline Weight & $5.20 \mathrm{~kg}$ & $1.00 \mathrm{Kg}$ & $5.35 \mathrm{~kg}$ & $9.70 \mathrm{~kg}$ \\
\hline Cost & $€ €$ & $€$ & $€ € €$ & $€ € € €$ \\
\hline
\end{tabular}

Table 1 Main characteristic of the employed Terrestrial Laser Scanners.

\subsection{Data acquisition strategy and comparison}

Before the acquisition, in order to adopt a common reference system for all the acquisitions performed, a set of 12 high reflectivity markers was positioned and measured using a Total Station following a traditional topographic approach.

A number of five scans (one in each corner and one in the middle of the room) was acquired inside the "Green Room" with each laser scanner, in order to guarantee the coverage of all the surfaces of the room with the lower possible loss of data due to occlusions and recesses. The only exception is represented by Riegl VZ-400i: in this case, a higher number of scans with an oblique orientation was needed to achieve a complete coloured point cloud, because of the limited FoV (Field of View) of the laser and the embedded camera. Therefore, six more scans with oblique $\left(45^{\circ}\right)$ configuration of the laser scanner have been required to acquire also the surfaces of the vaulted ceiling
(Figure 1) and to ensure adequate overlapping for the subsequent process of scan registration.

The acquisition time has been monitored for each deployed system. The number of scans required to acquire all the room features and the minutes needed by each sensor for a single acquisition (both point cloud and image acquisition phases) are reported in Table 2:

\begin{tabular}{|c|c|c|}
\hline Laser Scanner & $\mathrm{n}^{\circ}$ required scan & $\begin{array}{c}\text { required time } \\
\text { [minutes/scan] }\end{array}$ \\
\hline Faro Focus 3D X 330 & 5 & ca 9 \\
\hline Leica BLK360 & 5 & ca 5-6 \\
\hline Leica RTC360 & 5 & ca 5-6 \\
\hline Riegl VZ-400i & 11 & ca 10-12 \\
\hline
\end{tabular}

Table 2. Number of scans required to acquire all the surfaces of the "Green Room" and approximate time needed for each sensor for a single acquisition. 

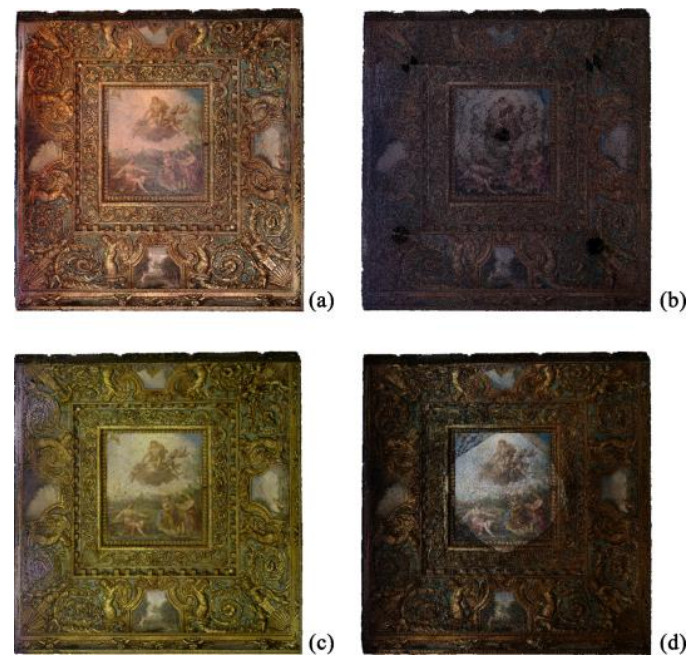

Figure 1. Point clouds of the vault of the "Green Room" acquired with (a) Faro Focus3D X 330, (b) Leica BLK 360, (c) Leica RTC360 and (d) Riegl VZ-400i.

As it is possible to observe, the fastest acquisitions have been performed by two Leica systems (BLK360 and RTC360). It is also worth mentioning that these two systems are the ones characterised by the higher portability among the four tested. Faro Focus ${ }^{3 \mathrm{D}}$ X 330 and Riegl VZ-400i are instead the sensors that required a longer time for a single acquisition (Table 2), however, the first one has been able to acquire a more significant portion of the room, due to its higher FoV. Therefore, considering the number of scans needed to acquire the entire room using both sensors, the overall time required by Faro sensor has been approximately 45-50 minutes while for Riegl it was around 2 hours.

\begin{tabular}{|c|c|c|}
\hline Laser scanner & $\begin{array}{c}\text { N. points/scan } \\
\text { (average value) }\end{array}$ & $\begin{array}{c}\text { File size }[\mathrm{MB}] \\
\text { (average value) }\end{array}$ \\
\hline Faro Focus ${ }^{3 \mathrm{D}}$ X 330 & ca $28,000,000$ & ca 700 \\
\hline Leica BLK360 & ca $49,000,000$ & ca 1,200 \\
\hline Leica RTC360 & ca $164,000,000$ & ca 4,200 \\
\hline Riegl VZ-400i & ca 71,000,000 & ca 1,800 \\
\hline
\end{tabular}

Table 3. Numbers of points for each scan (average value) and file size (average value).

After the processing phase of the different LiDAR datasets, that was completed following consolidated approaches and using ad hoc solutions for each system; various analyses were achieved. These analyses were aimed at evaluating the main differences among the different datasets and their peculiar characteristics and for understanding the advantage and disadvantage of the tested sensors used for a survey related to a restoration project.

The first investigated aspect was related to the density. The analysis was carried out (by mean of the number of neighbors approach), on different areas segmented from the point clouds of each laser, using the open-source software Cloud Compare. Although the point clouds acquired with Riegl have less than half of the number of points of Leica RTC360 scan (Table 3), their densities are comparable, as it can be observed in Figure 2 (ca $75 \mathrm{pts} / \mathrm{V}$ sphere $\mathrm{r}=1 \mathrm{~cm}$ for Riegl and ca $94 \mathrm{pts} / \mathrm{V}$ sphere $\mathrm{r}$ $=1 \mathrm{~cm}$ for Leica RTC360). This aspect is mainly related to the limited FoV of Riegl sensor $\left(100^{\circ} \times 360^{\circ}\right)$, as previously reported, that causes a lack of data over the upper part of the scanner. The point cloud with the lower density appears to be the one obtained using Faro Focus ${ }^{3 \mathrm{D}}$ X 330 . However, it needs to be specified that the acquisition has been achieved using a resolution parameter set of $1 / 5$ (equivalent to a resolution of 1 $\mathrm{pt} / 8 \mathrm{~mm}$ at $10 \mathrm{~m}$ of distance). According to this setting, the acquisition time is of ca 9 minutes. Increasing these parameters allows to obtain a denser point cloud, but with a relatively high impact on the acquisition time (using higher settings the acquisition of a single scan would have required from ca 11 minutes up to ca 2 hours at the highest resolution). The adopted parameter setup has been therefore considered a fair compromise between acquisition time and point cloud density.

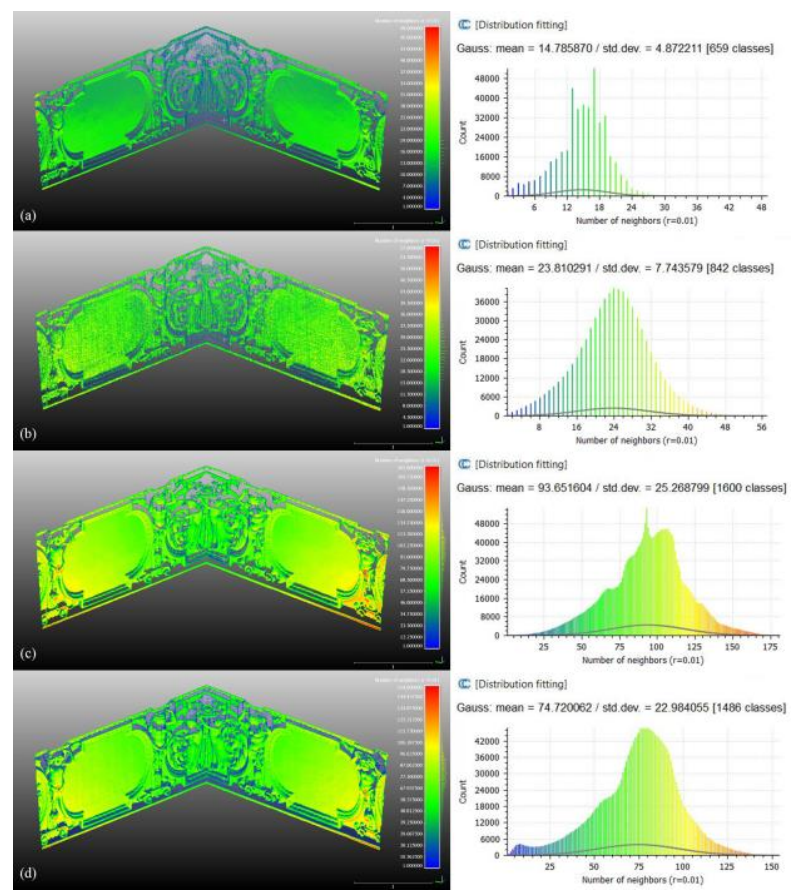

Figure 2. Density comparison between the adopted laser scanners (number of neighbour analysis, pts $/ \mathrm{V}$ sphere $\mathrm{r}=1 \mathrm{~cm}$ ) (a) Faro Focus3D X 330, (b) Leica BLK360, (c) Leica RTC360, (d) Riegl VZ-400i.

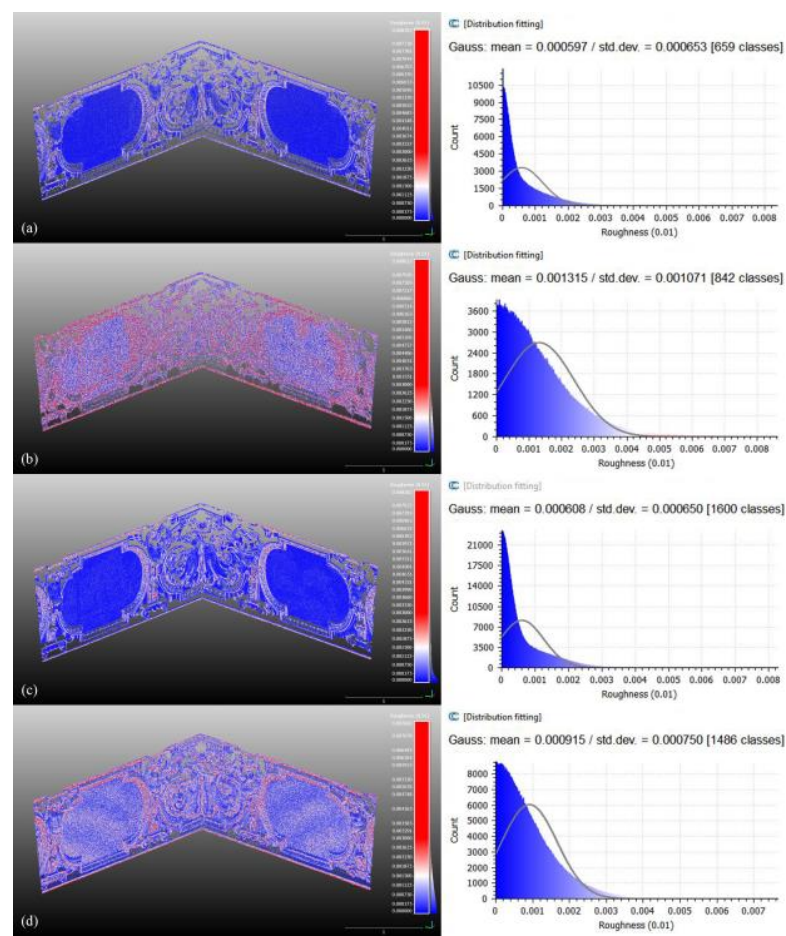

Figure 3. Roughness index maps of the adopted laser scanners (roughness analysis, di/V sphere $\mathrm{r}=1 \mathrm{~cm}$ ). (a) Faro Focus3D X 330, (b) Leica BLK360, (c) Leica RTC360, (d) Riegl VZ-400i. 
The second performed analysis was related to the roughness in order to evaluate the magnitude of the noise for each scan. Also, in this case, the Cloud Compare software has been used. The tool implemented in the software measures for each point the distance between the points and the best fitting plane computed on its nearest neighbours (located inside a sphere with a radius selected by the user, in this case, $1 \mathrm{~cm}$ ). As it can be observed in Figure 3, data derived from Leica RTC360 and Faro Focus ${ }^{3 \mathrm{D}}$ X 330 show comparable roughness values and are the ones with the lowest noise values among the four datasets. On the other hand, the point cloud characterised by higher values of roughness appears to be the one obtained from BLK360, followed by the one acquired with Riegl VZ-400i.

Moreover, after completing the analyses on the point clouds acquired with the different sensors, further tests were completed for the generation of a polygonal mesh for each dataset, in order to perform a comparison also between this type of 3D models. The point clouds have been imported in the photogrammetric software Agisoft Metashape to exploit the automatic mesh generation tool implemented in the software. During previous research experiences (Spanò et al. 2018), it has been observed that the performance of the algorithms of the meshing tool implemented in Metashape are extremely powerful, especially in noise mitigation. Moreover, another aim was to obtain the 3D mesh with a semi-automatic procedure to use similar parameters and thus to achieve a more objective comparison between the different results. Before the step of mesh computation, it was necessary to recompute the point cloud normal. This step is crucial since it has been observed that the import of LiDAR dataset into this photogrammetric software can lead to some errors in normals computation if an a priori check is not performed. After converting the point clouds to .ply format, they have been imported in the open-source software Meshlab. The filter "Compute normals for points sets" has been applied: for each point, a best-fitting plane is interpolated considering a number of closest points (the number is selected by the user) and normals are computed in respect to that plane.

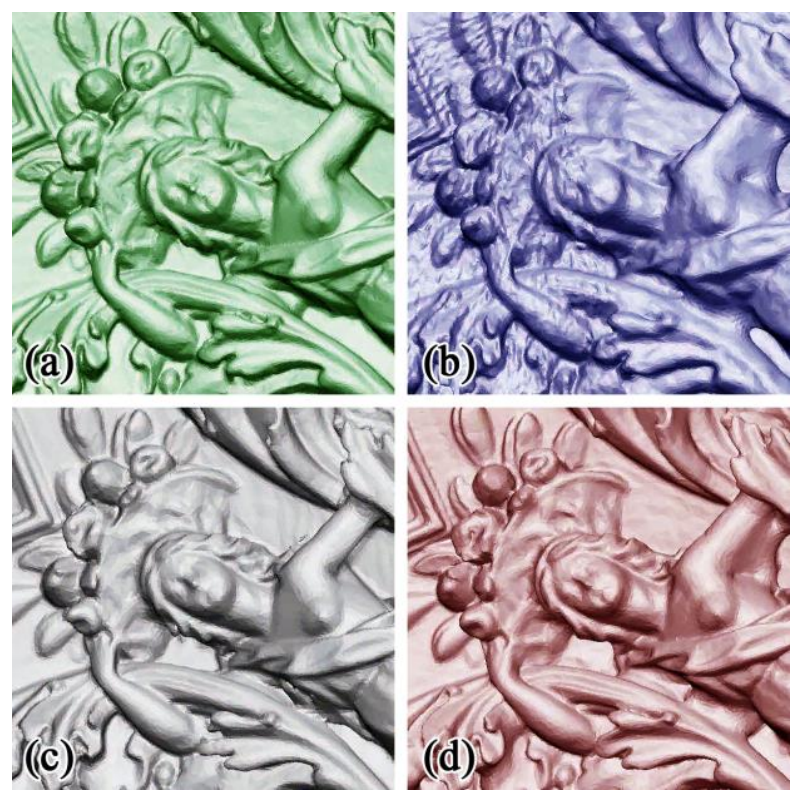

Figure 4. Particular of the meshes of the vault generated from the four datasets. (a) Faro Focus3D X 330, (b) Leica BLK360, (c) Leica RTC360, (d) Riegl VZ-400i.

The mesh that presents the highest noise is the one generated from Leica BLK360 dataset (Figure 4b), characterised by a lower level of detail, coherently with the results of the roughness analysis performed on the original point clouds. The meshes obtained from the other datasets show a comparable level of detail. A specific consideration could be addressed on the 3D model achieved from the Riegl data: in some areas (e.g. as is possible to observe under the left arm of the female figure stucco decoration visible in Figure 4d) the achieved model has some topological errors due to lack of data caused by noise (in the proximity of occlusions or recesses).

\section{IMAGE BASED SURVEY}

As is well known, the analyses of the decay and the different pathologies that can affect a palimpsest like the one of the Green Room is a crucial step before a restoration project. The radiometric component embedded in the metric products is thus of prior importance. In general, the RGB camera equipped in TLS systems presents a low-medium resolution and as a consequence the quality of the achievable radiometric products is not suitable for accurate analysis like the one required by the restorers. To overcome this issue, a complete photogrammetric survey was planned and performed as well, to guarantee the availability of high-resolution RGB information in the final derived products. The image acquisition phase was completed using two different sensors: a DSLR (Digital single-lens reflex) camera and a small UAV (Unmanned Aerial Vehicle) platform. The employed camera was a Canon EOS 5DS R, equipped with a $25 \mathrm{~mm}$ Zeiss lens while the UAV was an Anafi by Parrot.

The acquisition with the DSLR was completed following typical approaches (high overlapping, integration of images with different attitude) and a total of 195 images were acquired with an average estimated GSD of $0.9 \mathrm{~mm}$. To obtain the best results using this approach, it was also decided to use a set of led panel lights that was moved during the acquisition phase to achieve a homogenous illumination of the decorative surfaces. This aspect was particularly challenging during the acquisition phase, and a photographic tripod in combination with the remote control of the camera was used as well. Concerning the UAV system, the main objective was to acquire the floor of the "Green Room" from a proper acquisition distance, maintaining a nadiral camera configuration. Thanks to the Anafi it was possible to fly in the room safely and to acquire a total of 36 images from an elevation of about 4 meters (estimated GSD: 0.2 $\mathrm{mm}$ ). For the processing of the images a standard photogrammetric approach was followed inside the commercial software solution Agisoft Metashape, main details of the processed dataset are reported in Table 4.

\begin{tabular}{|c|c|c|}
\hline N. Images & Tie points & Dense cloud \\
\hline 231 & 412,903 & $166,874,750$ \\
\hline
\end{tabular}

Table 4. Main details of the photogrammetric process.

Thanks to the overlapping between the aerial and terrestrial dataset, that was projected and guaranteed during the acquisition, it was possible to coregister and process together the two datasets of images. Following the traditional pipeline, a set of control points was used during the processing to assess the metric accuracy of the overall operation. For the processing 9 points were measured on the field by mean of traditional topographic approach, 6 of them were used as Ground Control Points and 3 as Check Points. The mean RMSE is millimetrelevel (between 2 and $3 \mathrm{~mm}$ ) for both the GCPs and CPs.

As is possible to see from Figure 5, it was possible to obtain a complete, high detailed 3D model of the green room. This model, in combination with the ones provided by the different employed TLS systems, can be the base to project future restoration projects, being able to guarantee the creation of a series of added-values products. 


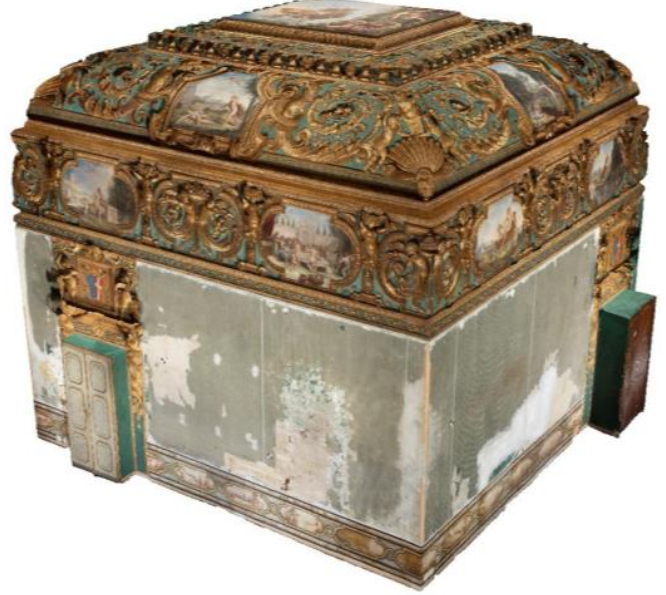

Figure 5. Side view of the photogrammetric model generated from the combination of the aerial and terrestrial dataset.

\section{FIRST CONCLUSION AND FUTURE PERSPECTIVES}

During these first steps of the reported research experience, four terrestrial LiDAR systems have been tested, and the acquired point clouds have been analysed to evaluate the different performances and output of the sensors.

As regards the acquisition phase, Leica BLK360 and Leica RTC360 proved to be the solution with higher portability; also, the acquisition performed with these sensors is the faster between the four tested laser scanners. On the contrary, Riegl VZ-400i is the less manoeuvrable device (due to its relatively high weight) and, because of the limited FoV, it requires a more significant number of scans (in comparison to other solutions) to cover the same surfaces. According to the characteristic mentioned above, and thanks to its very long acquisition range (800 $\mathrm{m})$, the Riegl laser scanner appears to be more suitable for outdoor applications.

The less performing point cloud in terms of noise and level of detail, as revealed by the performed analyses (the results of which can be observed in the previous sections), is the one acquired using Leica BLK360. However, during the tests, the Leica BLK360 has achieved excellent results in terms of portability and acquisition rapidity. Also, it represents a very competitive solution in terms of cost due to its relatively low market price (the smaller between the used LiDAR systems).

Finally, as observed in the roughness analysis presented before, the point clouds acquired from Leica RTC360 and Faro Focus ${ }^{3 \mathrm{D}}$ X 330 are those less affected by noise. Generally, it should be underlined that all the used systems allowed to obtain an accurate geometric description of the elements of the "Green Room" with a level of detail which can be considered sufficient for most of the current professional and research applications. This aspect is even more evident when a knowledge process related to the restoration process needs to be followed. In this case it is essential to merge a high accurate geometric definition with decay, cracks and material information that could be only provided with a complete radiometric representation. In this scenario, the geometric definition obtained from LiDAR systems contributes to adequately describe the geometrical features of the room while it has been observed that the photogrammetric approach is preferable as regards the radiometric contents (thanks to the orthoimagery generation).

Orthoimagery represents a typical metric product and nowadays became a fundamental support to the restoration operations. For this reason, it is recommended, when possible, to integrate
LiDAR scans with photogrammetric data for orthoimagery generation (that allows considering in detail the material consistency on the examined object - Figure 6). It is important to underline as well that nowadays not only typical 2D representations are requested when a restoration project is performed; even more, the possibility of 3D model visualization are exploited from the actors involved in the process in order to consider the whole object during the analysis from a nontraditional point of view.

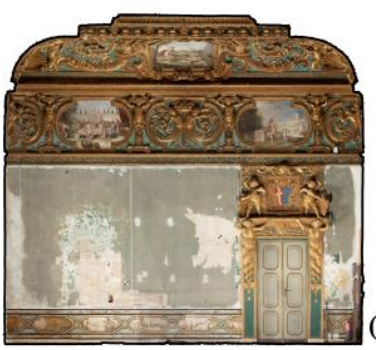

(a)

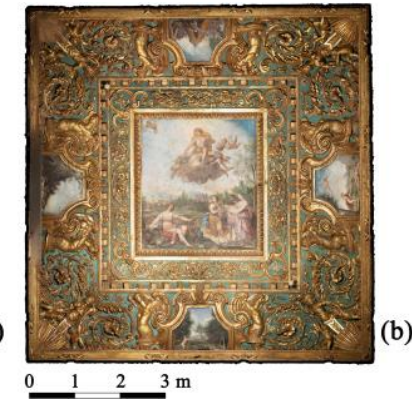

$1 \quad 2 \quad 3 \mathrm{~m}$

Figure 6. (a) "Green Room" western wall orthoimage (obtained from photogrammetric dataset). (b) Green Room vaulted ceiling orthoimage (obtained from photogrammetric dataset).

Considering the analyses that have been reported in the previous sections, in connection with the obtained results, two main future perspectives have been considered. The first one is connected to HBIM models generation starting from realitybased data, as a tool able to potentially manage several issues related to these types of restoration operations. The second is related to the enrichment of 3D models and orthoimagery with spectral information, following a data fusion strategy that would allow in-depth analyses for the conservation status of built heritage (for example as concerns decay detection in the nonvisible light spectrum).

\subsection{HBIM and restoration}

The increasing interest in the use of parametric models associated to a BIM environment is under development in several disciplines related to the architectural studies, e.g. urban design, architectural design, etc., and will probably become a standard in the next years. The same phenomena are also happening in the conservation and restoration areas. According to this growing attention is interesting to reflect and investigate how the high-detailed 3D models (very dense point clouds or triangulated mesh) carried out from range-based or image-based techniques could be used for parametric modelling purposes, especially when is necessary to deal with an articulated decorative scheme like the one of the "Green Room".

HBIM represents one of the most promising tools for information management and in the last few years the potentialities connected to this technology are emerging in the framework of restoration project (Bruno and Roncella 2019; Brumana et al. 2017); however, it is necessary to continuously develop new strategies in order to face the problems related to interoperability between this systems and the very accurate and complex reality-based models that it is possible to achieve nowadays. One of the limitations of these reality-based models is represented by the issues connected to data management in many BIM platforms. These issues are often caused by the dimension of the file associated with this reality-based models and with the complexity of their topology.

Due to the lack of advanced modelling tools in many BIM applications, that provide management tools for new buildings rather than pre-existing heritage assets (Banfi 2020), frequently 
a substantial simplification of the acquired geometries is required. This aspect is a crucial point since in the framework of cultural heritage documentation, a high level of detail is not only recommended but almost mandatory, due to the necessity to accurately and adequately study the shapes, geometries, features and irregularities of the researched objects.

In recent years many researchers working in the field of Geomatics for conservation and valorisation investigated new strategies connected with these issues (Banfi 2020). Many software dedicated to $3 \mathrm{D}$ modelling has recently implemented automatic shape recognition tools, allowing to perform primitive geometries extraction and minimizing the discrepancies using the least-squares method. Currently, there are many commercial solutions as regards the identification of simple shapes (as planes, in case of walls or planar elements, or spheres, cylinders, cones and tubular shapes - Figure 7).

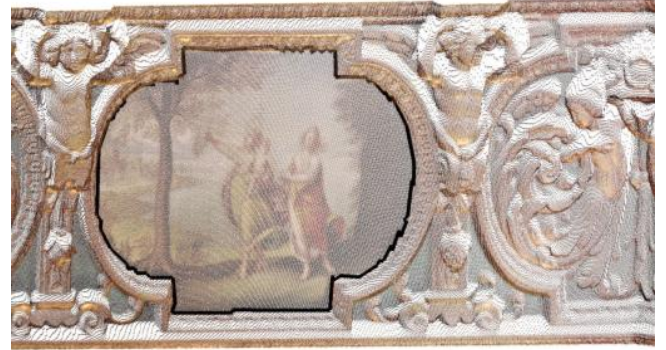

Figure 7. Example of automatic extraction of a planar surface from a LiDAR point cloud using 3DReshaper platform.

However, in case of complex surfaces (such as articulated stucco decorations visible on the vaulted ceiling of the "Green Room"), it is complex to automate the process of geometries extraction. In this case, a solution can be represented by free form reverse modelling techniques (Diara and Rinaudo 2019), like NURBS (Non-Uniform Rational Basis Spline) by mean of specific tools such as McNeel Rhinoceros, that provides advanced modelling approaches to perform accurate geometry reconstruction. However, even this strategy presents several issues, since to carry out these reconstruction processes (which are extremely time spending) a high degree of expertise is needed by the operator. Moreover, NURBS modelling software (like McNeel Rhinoceros or Autodesk 3DStudio Max) are nonparametric software, and the modelled geometries require further parametrisation processes (using specific plug-ins or other solutions) (Tommasi et al. 2016).

During this research experience, some tests and modelling attempts using the strategies mentioned above are currently in progress in order to identify the most efficient and effective strategy to achieve a model suitable for the intended purposes. The performed tests deal with two main aspects. The first one is related to the parametric model of the "Green Room" that was achieved without any problem following the geometry easily extracted from the laser scanner point clouds (an example of the vault is reported in Figure 8). The second part, that is still under analysis, is related to the geometric description of the elaborate stucco decorations of the room that is crucial for a correct representation of the consistency of the studied area. A suitable solution is related to the free-form modelling strategy (Figure 9) which deal with some disadvantage since it is a very time spending approach and moreover require highly experienced operators. This solution is, as a consequence, not useful for large areas like the ones that are located in the analysed area, but also in the other rooms of the Valentino Castle and in general in most of the architectural heritage.

In the next years, this research sector will probably face development of the automatisation degree of the previously described strategies, representing a challenge indeed also for researchers operating in cultural heritage valorisation fieldwork. Another aim will be to make these tools accessible to a broader audience (and not only to specialists), without sacrificing accuracy criteria and a high level of detail of the models.

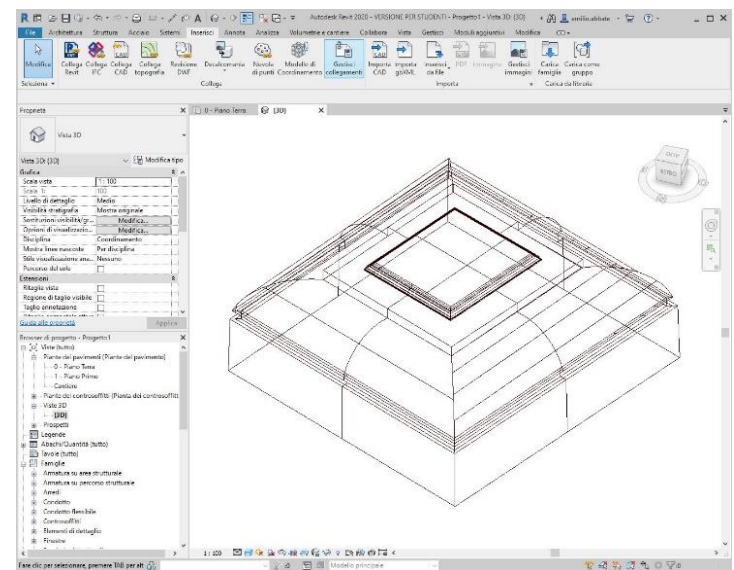

Figure 8. Simplified 3D model of the vault imported inside parametric software Autodesk Revit.

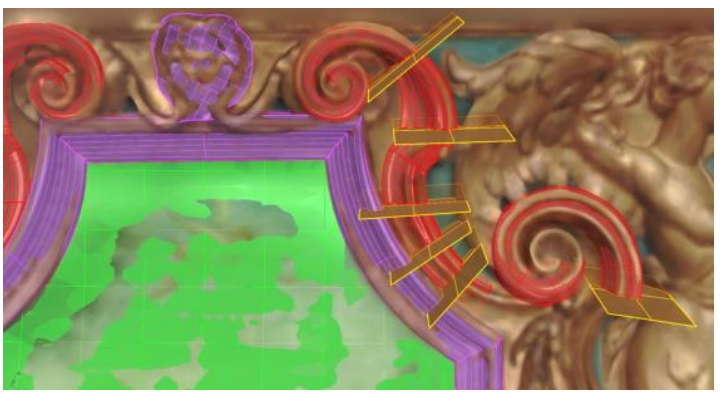

Figure 9. Example of reverse modelling starting from 3D mesh in Rhinoceros platform.

\subsection{Integration between traditional RGB datasets and hyperspectral images (co-registration strategies)}

Finally, some tests were also performed concerning the possibilities connected to the developments of SfM (Structure from Motion) algorithms in the last years. These developments allow to process and extract information not only from traditional true colour digital photos but also from spectral images, performing investigations in the non-visible light spectrum. The use of these techniques is widespread in a high number application fields (Khaliq et al. 2018): among them precision agriculture, environmental monitoring, chemical analysis, medical research and, of course, cultural heritage documentation and investigation. As concerns the latter application, it is proved that thermal and spectral imaging can provide considerable support to the research community operating in the framework of valorisation and conservation (Pronti et al. 2019; Adamopoulos and Rinaudo 2020) and that they represent a valuable non-invasive diagnostic tool for monitoring invaluable assets belonging to our built heritage. Undoubtedly the use of these technologies constitutes an opportunity to develop researches for the conservation of the built heritage, and a useful tool for professionals operating in the restoration field.

For example, hyperspectral imaging techniques allow associating to every pixel of the acquired image the spectral content of the scene to obtain the so-called "hyperspectral cube" where the spatial information is combined with the spectral components in each pixel of an image (Zucco et al. 2017; 
Padoan et al. 2008). Through spectrum technologies, it is possible to analyse the surfaces of the investigated objects to detect deterioration/decay on masonries or frescoes ( $\mathrm{Lu}$ et al. 2015).

An exciting development could be represented by the opportunity to overlay the different information by integrating data acquired with various sensors and techniques. In the test case presented in this research, the information contained in hyperspectral images has been combined with more accurate geometries derived from a optical dataset, which is characterised by a higher resolution and level of detail.

Seven hyperspectral images of the western wall of the Green Room have been collected following overlapping photogrammetric criteria (overlapping ca 70-80\%; selected resolution of the photos: 1010 x 1010 pixels; spectral range: wavelength from $500 \mathrm{~nm}$ to $900 \mathrm{~nm} ; 197$ bands) using a hyperspectral camera (Hyperspectral frame Rikola produced by Senop, Musci et al. 2019). After the acquisition, the images have been processed with the software Agisoft Metashape.

The followed approach consists in co-registration between datasets the one acquired by the Rikola ( 7 images) and the one obtained by the DSLR (Digital Single Lens Reflex) camera (195 images - Figure 10).

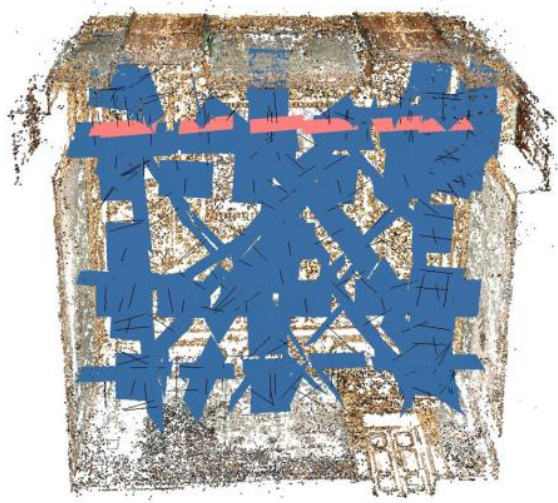

Figure 10. Co-registration between optical dataset (blue) and hyperspectral dataset (light orange).

According to this strategy, the dense cloud generation and 3D mesh processing have been performed using only the true colour images, which are characterised by the highest spatial resolution (disabling hyperspectral images, to achieve a more detailed and topologically correct model). Finally, the orthomosaic generation has been performed using only the hyperspectral dataset (Figure 11). In this way, the orthophoto generation has been achieved using high-resolution 3D data (Digital Surface Model) generated from DLSR images.

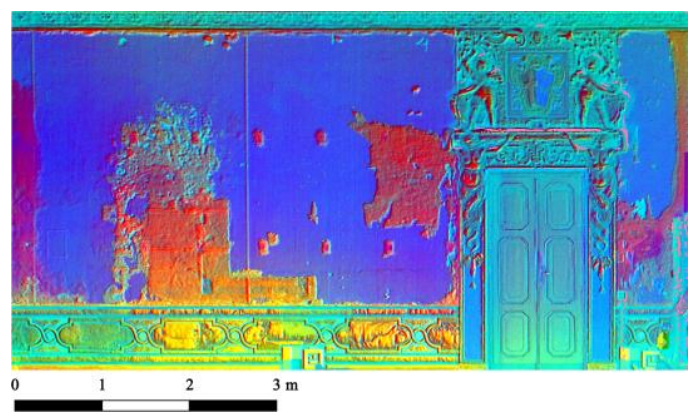

Figure 11. Hyperspectral orthomosaic of the western wall of Green Room displayed on ENVI platform (false colour representation from PCA (Principal Components Analysis) of the multi-band raster).
Also in this case a set of 9 points has been used to provide GCPs (6) and CPs (3) and the observed mean RMSE is coherent with the results observed in the previous sections (between 2 and $3 \mathrm{~mm}$ ).

One of the advantages of the followed strategy is represented by the opportunity to perform co-registration of single hyperspectral images. Following this workflow, it is not necessary to carry out multiple acquisitions with a hyperspectral camera observing overlapping criteria since it is possible, to perform directly a co-registration between true colour datasets and a single hyperspectral acquisition (Figure 12). This procedure can save time during the acquisition phase since the traditional photogrammetric survey techniques involving the use of a DSLR camera are generally more rapid than hyperspectral acquisitions. In addition, this approach reduces the need to establish distinct GCPs in hyperspectral images.
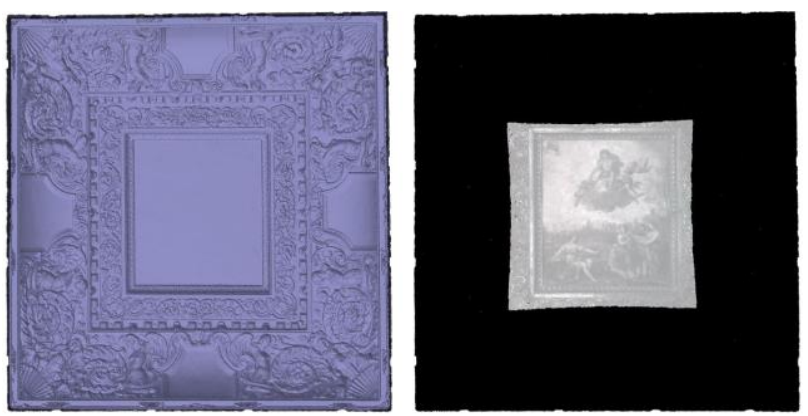

Figure 12. Example of single hyperspectral image of the vaulted ceiling co-registrated with true colour dataset.

The described workflow represents a very interesting perspective as regards the potentiality to merge data from different sources both for orthophoto generation and 3D visualisation for integrating and manage information of different nature. In addition, it should be underlined the importance of the opportunity connected to the development of new strategies in order to carry out further analyses for diagnostic purposes using a non-invasive contactless methodology, like the ones offered by the constant improvement of spectrum technologies. As well known to the restoration community, heritage assets are intrinsically fragile, and it is therefore necessary to continuously assess new tools, instruments and techniques that take account of that vulnerability and that frailness which characterises our historic buildings. A challenge for the future is, for sure, represented by the possibility to develop further investigations moving in this direction. For this reason, constant and attentive documentation can be an answer, to improve the resilience of cultural heritage assets and efficiently monitoring them.

\subsection{Final reflexions}

The experience presented in the current work has underlined the necessity, in the framework of heritage documentation, to focus about the connection between the requirements of the survey and the techniques with which the data are acquired. Considering the LiDAR approach, which represents a consolidated and reliable solution, a wide variety of systems is nowadays commercially available with very different technical features, strengths and weaknesses. Therefore, the data acquisition should be attentively planned choosing the instrument based on the requirements of the survey. Another essential aspect that must be considered is related to the economic sustainability of the adopted solutions In this sense the photogrammetric approach represents not only a reliable 
low-cost alternative but, as evidenced in the previous sections, also the best solution for othoimagery generation which represents a fundamental resource in the fieldwork of restoration.

Furthermore, the scenarios opened by the use of BIM systems in the framework of heritage have been considered. How previously stressed, the potential of this technology is exciting and it has allowed achieving remarkable results. However, the difficulties connected to the production of models with high accuracy and high level of detail in addition to the issues related the automatisation of the modelling process are is the real bottleneck of the BIM software. These aspects still constitute a limitation and a challenge for the next future where without any doubt the cultural heritage documentation will be even more carried out using HBIM solutions

Finally, it should be mentioned how the possibilities offered by the progress of new image-based sensors can provide valuable support in developing new representations and analyses of not visible features. However, several issues still have to be considered, mainly connected to the hyperspectral sensors (camera calibration, radiometric calibration, ecc.) and to the use of the acquired data (for example the identification of the right bands for the decay detection or cracks analysis), which are yet unaddressed in the current paper and still under depth analysis.

\section{ACKNOWLEDGEMENTS}

The authors would like to acknowledge the interdepartmental centre of the Politecnico di Torino PIC4SER (Politecnico Interdipartmental centre for service robotic) to borrow the Rikola Hyperspectral camera. The authors would also like to express their gratitude to Prof. F. Giulio Tonolo and M. A. Musci for their precious help during hyperspectral images acquisition and processing.

\section{REFERENCES}

Adamopoulos, E., Rinaudo, F. (2020). Enhancing Image-Based Multiscale Heritage Recording with Near-Infrared Data. International Journal of Geo-Information, 9(4), 269.

Bacci, G., Bertolini, F., Bevilacqua, M. G., Caroti, G., MartínezEspejo Zaragoza, I., Martino, M., Piemonte, A. (2019). HBIM methodologies for the architectural restoration. The case of the exchurch of San Quirico all'Olivo in Lucca, Tuscany. ISPRS International Archives of the Photogrammetry, Remote Sensing and Spatial Information Sciences, XLII-2/W11, 121-126.

Banfi, F. (2020). HBIM, 3D drawing and virtual reality for archaeological sites and ancient ruins. Virtual Archaeology Review, 11(23), 1-18.

Beltrami, C., Cavezzali, D., Chiabrando, F., Iaccarino Idelson, A., Patrucco, G., Rinaudo, F. (2019). 3D digital and physical reconstruction of a collapsed dome using SfM techniques from historical images. ISPRS - International Archives of the Photogrammetry, Remote Sensing and Spatial Information Sciences, XLII-2/W11, 217-224.

Brumana, R., Della Torre, S., Oreni, D., Previtali, M., Cantini, L., Barazzetti, L., Franchi, A., Banfi, F. (2017). HBIM challenge among the paradigm of complexity, tools and preservation: the Basilica di Collemaggio 8 years after the earthquake (L'Aquila). ISPRS - International Archives of the Photogrammetry, Remote Sensing and Spatial Information Sciences, XLII-2/W5, 97-104.

Bruno, N., Coïsson, E., Diotri, F., Ferrari, L., Mikolajewska, S., Morra di Cella, U., Roncella, R., Zerbi, A. (2019). History, geometry, structure: interdisciplinary analysis of a historical bridge. ISPRS - International Archives of the Photogrammetry, Remote Sensing and Spatial Information Sciences, XLII-2/W11, 317-323.
Bruno, N., Roncella, R. (2019). HBIM for Conservation: A New Proposal for Information Modeling. Remote Sensing, 11(15), 1751.

Chiabrando, F., Sammartano, G., Spanò, A. (2016). Historical building models and their handling via 3D survey: from point clouds to user-oriented BIM. ISPRS - International Archives of the Photogrammetry, Remote Sensing and Spatial Information Sciences, XLI-B5, 633-640.

Diara, F., Rinaudo, F. (2019). From reality to parametric models of Cultural Heritage assets for HBIM. ISPRS - International Archives of the Photogrammetry, Remote Sensing and Spatial Information Sciences, XLII-2/W5, 413-419.

Girelli, V. A., Tini, M. A., Dellapasqua, M., Baitelli, G. (2019). High resolution 3D acquisition and modelling in Cultural Heritage knowledge and restoration projects: the survey of the Fountain of Neptune in Bologna. ISPRS - International Archives of the Photogrammetry, Remote Sensing and Spatial Information Sciences, XLII-2/W11, 573-578.

García-León, J., Collado Espejo, P. E., Jiménez González, F. J. (2019). Negro Tower: documentation, conservation, and restoration. ISPRS - International Archives of the Photogrammetry, Remote Sensing and Spatial Information Sciences, XLII-2/W15, 489-496.

Khaliq, A., Musci, M. A., Chiaberge, M. (2018). Analyzing relationship between maize height and spectral indices derived from remotely sensed multispectral imagery. IEEE Applied Imagery Pattern Recognition Workshop (AIPR), Washington, DC, USA, 1-5.

Lu, D., Wang, Z., Zhang, D., Sun, M. (2015). System for hyperspectral data analysis, visualisation and fresco deterioration detection. 2015 IEEE International Conference on Multimedia Big Data, 312-317.

Musci, M. A., Aicardi, I., Dabove, P., Lingua, A. M. (2019). Reliability of the geometric calibration of an hyperspectral frame camera. ISPRS - International Archives of the Photogrammetry, Remote Sensing and Spatial Information Sciences, XLII-2/W13, 1701-1707.

Padoan, R., Steemers, A.G., Klein, M.E., Aalderink, B. J., de Bruin, G. (2008). Quantitative hyperspectral imaging of historical documents: technique and applications. Art Proceedings, 25-30.

Pracchi, V., Barazzetti, L. (2019). On the relationship between Geomatics and Conservation: lessons learned from integrated restoration laboratories. ISPRS - International Archives of the Photogrammetry, Remote Sensing and Spatial Information Sciences, XLII-2/W11, 981-988.

Pronti, L., Romani, M., Verona-Rinati, G., Tarquini, O., Colao, F., Colapietro, M., Pifferi, A., Cestelli-Guidi, M., Marinelli, M. (2019). Post-Processing of VIS, NIR, and SWIR Multispectral Images of Paintings. New Discovery on the The Drunkenness of Noah, Painted by Andrea Sacchi, Stored at Palazzo Chigi (Ariccia, Rome). Heritage, 2(3), 2275-2286.

Roggero Bardelli, C. (1992). Torino. Il Castello del Valentino. Lindau, Torino, IT.

Spanò, A., Chiabrando, F., Sammartano, G., Teppati Losè, L. (2018). Integrated imaging approaches supporting the excavation activities. Multi-scale geospatial documentation in Hierapolis (TK). ISPRS - International Archives of the Photogrammetry, Remote Sensing and Spatial Information Sciences, XLII-2. 1075-1082.

Tommasi, C., Achille, C., Fassi, F. (2016). From point cloud to BIM: a modelling challenge in the Cultural Heritage field. ISPRS International Archives of the Photogrammetry, Remote Sensing and Spatial Information Sciences, XLI-B5, 429-436.

Zucco, M., Pisani, M., Cavaleri, T. (2017). Fourier trasform hyperspectral imaging for cultural heritage. Fourier Transforms High-tech Application and Current Trends, INTECH: London, UK, 215-234. 